\title{
An Integrated Interactive Technique for Image Segmentation using Stack based Seeded Region Growing and Thresholding
}

\author{
Sirshendu Hore ${ }^{1}$, Souvik Chakraborty ${ }^{2}$, Sankhadeep Chatterjee ${ }^{3}$, Nilanjan Dey $^{4}$, \\ Amira S. Ashour ${ }^{5}$, Le Van Chung ${ }^{6}$, Dac-Nhuong Le ${ }^{7,8}$ \\ ${ }^{1}$ Department of Computer Science \& Engineering, Hooghly Engineering \& Technology College Chinsurah, India \\ ${ }^{2}$ Department of Computer Science \& Engineering, University of Kalyani, Kalyani, India \\ ${ }^{3}$ Department of Computer Science and Engineering, University of Calcutta, Kolkata, India \\ ${ }^{4}$ Department of Information Technology, Techno India College of Technology, Kolkata, India \\ ${ }^{5}$ Department of Electronics and Electrical Engineering, Faculty of Engineering, Tanta University, Egypt \\ ${ }^{6,7}$ Duy Tan University, Danang, Vietnam \\ ${ }^{8}$ Haiphong University, Haiphong, Vietnam
}

\begin{tabular}{l} 
Article Info \\
\hline Article history: \\
Received Mar 13, 2016 \\
Revised Aug 13, 2016 \\
Accepted Aug 23, 2016 \\
\hline
\end{tabular}

Keyword:

Image segmentation

Otsu's method

Region growing

Seed region growing method

Thresholding

\begin{abstract}
Image segmentation is a challenging process in numerous applications. Region growing is one of the segmentation techniques as a basis for the Seeded Region Growing method. A novel real time integrated method was developed in the current work to locate the segmented region of interest of an image based on the Region Growing segmentation method along with the thresholding supported image segmentation. Through the proposed work, a homogeneity based on pixel intensity was suggested as well as the threshold value can be decided via a variety of schemes such as manual selection, Iterative method, Otsu's method, local thresholding to obtain the best possible threshold. The experimental results were performed on different images obtained from an Alpert dataset. A comparative study wasarried out with the human segmented image, threshold based region growing, and the proposed integrated method. The results established that the proposed integrated method outperformed the region growing method in terms of the recall and F-score. Although, it had comparable recall values with that gained by the human segmented images. It was noted that as the image under test had a dark background with the brighter object, thus the proposed method provided the superior recall value compared to the other methods.
\end{abstract}

Copyright $@ 2016$ Institute of Advanced Engineering and Science. All rights reserved.

\section{Corresponding Author:}

Dac-Nhuong Le,

Haiphong University,

171 Phan Dang Luu, Kien An, Haiphong, Vietnam.

Email: nhuongld@hus.edu.vn

\section{INTRODUCTION}

Segmentation in imaging discipline is extremely valuable in numerous fields such as satellite imaging [1], computer vision [2] as well as medical applications in order to diagnose the abnormalities in the image [3]. It is an imperative step in every image analysis process. Image segmentation is considered as a key step for subsequent image representation/recognition phases of an image into more meaningful and easier representation to analyze [4]. It is defined as partitions of the spatial domain pixels into significant regions or parts that can be a complete specified object or a part of it. It is usually used to locate objects as well as boundaries (lines, curves, etc.) in images [5]. It provides none overlapping objects that labelled with different region numbers. Due to image segmentation, a segment set that collectively covers the whole image, or a set of contours extracted from the image is produced [6]. Each pixel in a region is analogous to some characteristics or computed property, such as color, texture, or intensity. Adjacent regions are extensively 
dissimilar with respect to the same characteristic(s). The main goal of segmentation is to clearly differentiate the object and the background in an image. Segmentation is considered to be a psycho-physical challenge, since it physically measured image features. The segmentation performance measurement depends on the associated meaning with the extracted regions. Simultaneously, the mathematical model of the extracted region is complicated as it requires some significance with respect to the given image. Consequently, segmentation is the main exigent problem as there is no generic technique has been developed yet for segmentation accurately with all kinds of images. This leads to certain assumptions, and attempts to develop the segmentation algorithms. According to the user interaction level requirements, the segmentation process can be classified into several techniques, which are manual segmentation, semi-automatic segmentation and automatic segmentation [7]. Manual segmentation is considered as the most accurate, but time-consuming. Semi-automatic segmentation involves the minimal user intervention to endow with a small amount of input and parameter to assist accurate segmentation. On the contrary, automatic segmentation is difficult to obtain accurate results as it does not engage any user input. Consequently, it is preferred with large number of images, but at the cost of accuracy. There are two main approaches in image segmentation, namely edge and region-based. Edge-based segmentation looks for discontinuities in the intensity of an image. Region-based segmentation looks for uniformity within a sub-regionbased on a desired property, e.g. intensity, color, and texture. Image segmentation techniques can also be classified into five approaches, such as Thresholdingbased method [8], Boundary-based method [9], Region-based segmentation method [10], Active contour model (Snakes) image segmentation [11], hybrid segmentation technique [12].

Since, Thresholding-based segmentation algorithms are efficient in terms of the computational complexity. Consequently, the current study is concerned with developing an interactively integrate system based on the Region Growing segmentation method along with the thresholding supported image segmentation. Thresholding methods are compulsory to convert an image to a binary image depending on the threshold values that extract the objects from the background.The organization of the remaining sections are as follows: Section 2 presents the concept of region growing image segmentation; Section 3 introduces the proposed system; Section 4 reports the results and discussion; finally the conclusion is depicted in Section 5.

\section{REGION GROWING IMAGE SEGMENTATION PROCESS}

Region based method is supported by the homogeneity principle that refers to the pixels with comparable properties, which clustered collectively to form a homogenous region [13]. This segmentation method is categorized into three types based on the principle of region growing as Region merging, Region splitting, and Split and merge. In addition to the three components of the region-growing method algorithm, which are the seed point selection, principle of growth, and terminating conditions.

The critical step in the region growing process is the seed point selection, which requires humancomputer interaction method as it can initially selected by the user based on various criteria and settles on the overall segmentation via the region growing technique. Whereas, the principle of growth states the pixel value of pixels in neighbour is less than the threshold [14]. Finally, the terminating conditions will prolong until it has no pixels remaining to complete the need of the principle of growth step. Thus, the Region growing method establishes with preliminary seeds and grows with neighbouring homogenous elements. The foremost intention of region growing is to map individual pixels (seeds) in the input image to a set of pixels named region, which was first initiated by Adams and Bischof in 1994 [15]. Accordingly, the user only must find a few representatives features, and then the region growing will locate all features of analogous properties in the same region. Thus, this leads to a semiautomatic segmentation process as it starts with an interactive seed point selection stage, followed by the region growing process. Two important criteria of region growing process are finding the homogeneity criteria and the threshold value selection.

\section{THE PROPOSED SYSTEM (Stack Based Seeded Region Growing Algorithm based threshold)}

The objective of this study was the development of an extended stack based region growing approach in [16] based on thresholding. Through the proposed system, a stack based data structure was used to traverse the neighbourhood pixels around the seed location to implement the two-dimensional (2D) seeded region grow algorithm. As the employment of the stack based data structure is straight forward, besides its capability in data access. The accomplishment of the region grow algorithm relies on: i) the initial seed selection, and ii) the appropriate criteria employed to terminate the recursive region grow process, which are the key in extracting the desired regions. On the whole, these criteria comprise object contrast with regard to background, region homogeneity, the region boundary strength, size, and consistency to the required texture features (e,g. texture, shape, color). Commonly, most of the region growing process is based on homogeneity criteria. In the proposed work, the selected homogeneity was based on pixel intensity. The algorithm was 
more or less the same, but the choice of homogeneity criteria was different. The difference between the current pixel and one of its neighbours has been calculated to find the pixel intensity. Then, if the value was within the threshold, then the pixel was part of the region. Although, the algorithm in [16] calculated the horizontal and vertical gradient of $3 \mathrm{X} 3$ sub-matrix, it divided it with $\mathrm{K}$ X maximum gradient presents in the image, and $\mathrm{K}$ is some predefined constant to control the region grow. The proposed work assumed region mean based homogeneity criteria, which have been calculated as follows:

a. Initialize region_mean with the pixel value of the seed point

b. Calculate new_region_mean $=$ (old_region_mean*no_of_points + pixel value of new entry) (no_of_points $+\overline{1}$ )

The region growing during the proposed algorithm implementation of 4-neighbours was illustrated in Figure 1. Since, the threshold value can be chosen in assorted ways as it depends on the image under test. Therefore, constant threshold for all the images can't be used. Then, local thresholdingwas used in this proposed study on an experimental basis as it endowed with better result. Subsequently, the proposed system was tested by applying various manual threshold inputs. Typically, the user can select the threshold based on some experimental basis, i.e. to choose the Otsu's, iterative, local or manual, to find the best possible threshold.A threshold value of 0.2 was fit for most of the tested images as proved after a series of experimental trials. In case of integrated process, local thresholding was used with structuring element disk. Other structuring elements were also tested, but the results obtained were unsatisfactory for most of the images.

\begin{tabular}{|c|c|c|}
\hline$x-1, y-1$ & $x, y-1$ & $x+1, y-1$ \\
\hline$x-1, y$ & $x, y$ & $x+1, y$ \\
\hline$x-1, y+1$ & $x, y+1$ & $x+1, y+1$ \\
\hline
\end{tabular}

Figure 1. Four Neighbours Considered for Region Grow

Similar pseudo code that used for computed tomography angiography image segmentation [16] was used in the current work for the stack based seeded region growing algorithm. The algorithm code is demonstrated as follows.

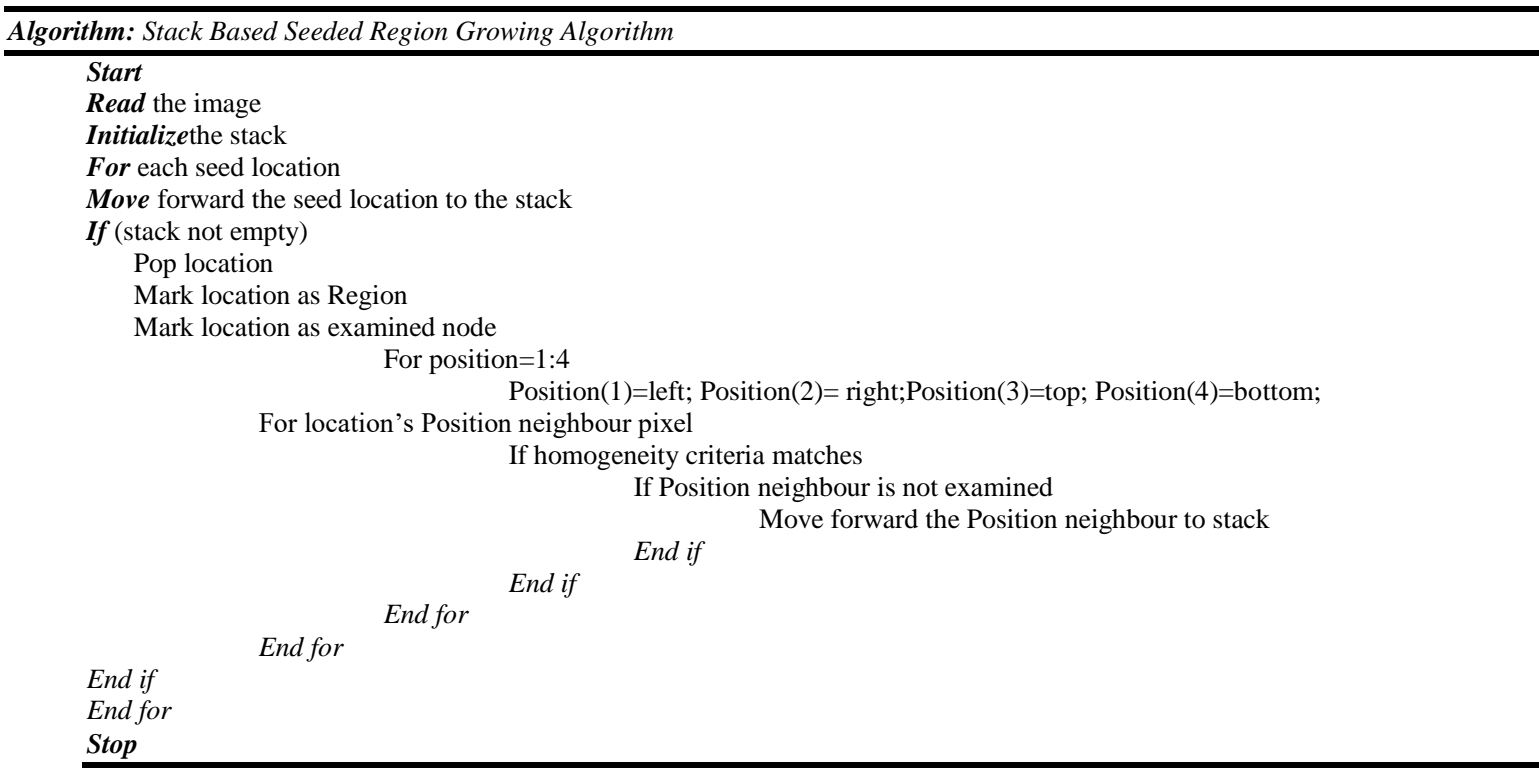

According to the steps above, the proposed system was illustrated in Figure 2. 


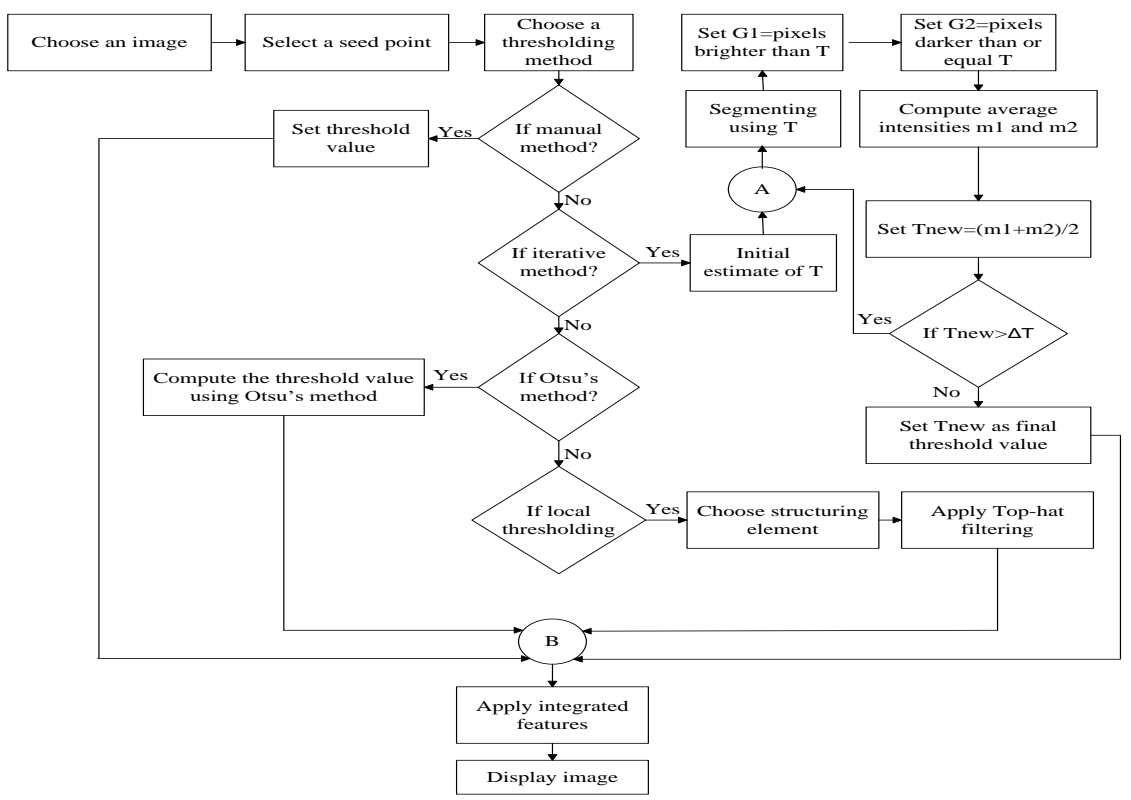

Figure 2. Schematic Diagram of Proposed Approach

As demonstrated in Figure 2, Otsu's method was applied to compute the threshold value. Due to the interaction step for seed initialization selection, the segmentation process becomes semiautomatic starting with an interactive seed point selection step, followed by the region growing process. The Weizmann institute of science database [17] is used for the experimental results. As a set of random images of different types of Alpert dataset which contains more than 100 images with a ground truth image called as human segmented image from [weizmann.ac.in] were used along with ground truth segmented image.

\section{RESULTS AND DISCUSSION}

A comparative study is accomplished to evaluate the proposedsegmentation system with different algorithms [18]. Figure 3 shows the results of eleven images as a sample of the original image, human segmentation (i.e. ground truth image that perceived by the human eye), region growing, and the proposed integrated approach.

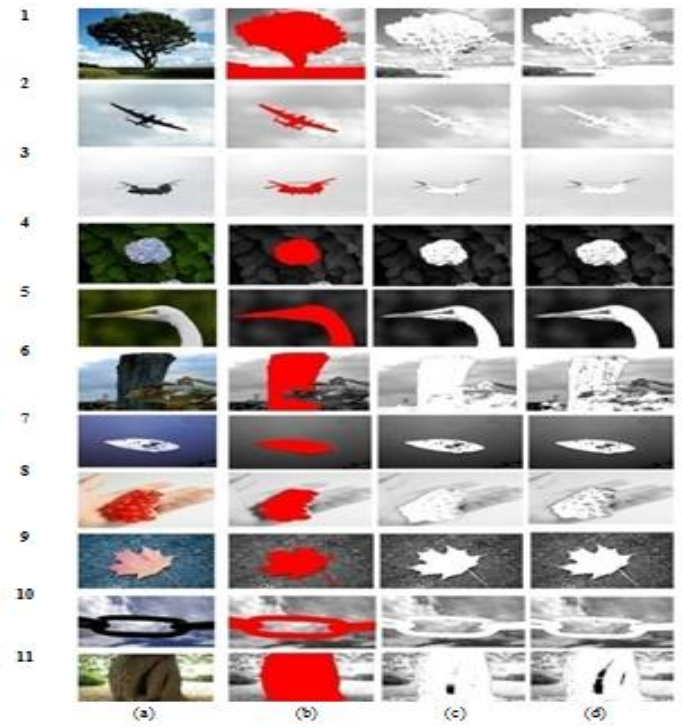

Figure 3. (a) Original Image, (b) Human Segmented Image, (c) Region Growing (0.2), (d) Integrated method 
Figure 3 depicts that the proposed integrated methods has superior segmentation results compared to the other approaches in Figure 3 (b) and (c).

In order to evaluate the proposed method performance compared to the other approaches, several metrics are measured, which defined as follows. The precision-recall metrics framework is both effective and intuitive and considered as a benchmark evaluation scheme in the information recovery area. Therefore, the recall, precision, and F-score are frequently used to evaluate the image segmentation performance process, where the objective is to detect boundary. The precision is defined as the probability that a resulting boundary pixel is a true boundary pixel [19]. The recall rate is known as the probability that a true boundary pixel is discovered [20]. In addition, the F-score is the harmonic mean of the precision and the recall [20], which is given by:

$$
\mathrm{F}=\frac{2 \times \text { Precision } \times \text { Recall }}{\text { Precision }+ \text { Recall }}
$$

where,

$$
\begin{aligned}
& \text { Precision }=\frac{\text { detectedtrueboundarypixels }}{\text { detectedboundarypixels }} \\
& \text { Recall }=\frac{\text { detectedtrueboundarypixels }}{\text { all trueboundarypixels }}
\end{aligned}
$$

Figures 4-9 show the comparative results of different algorithm with the proposed algorithm. Figures 4 and 5 illustrate the comparison of the F-score and the precision values; respectively for the human, region growing and integrated segmentation methods.

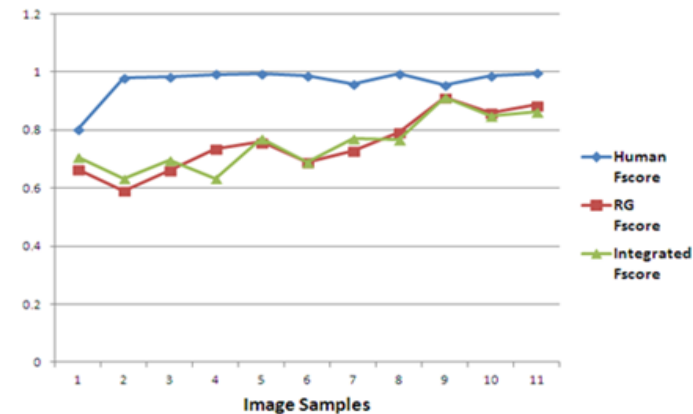

Figure 4. Comparison of the F-Score Value for the Human, Region Growing and Integrated Segmentation

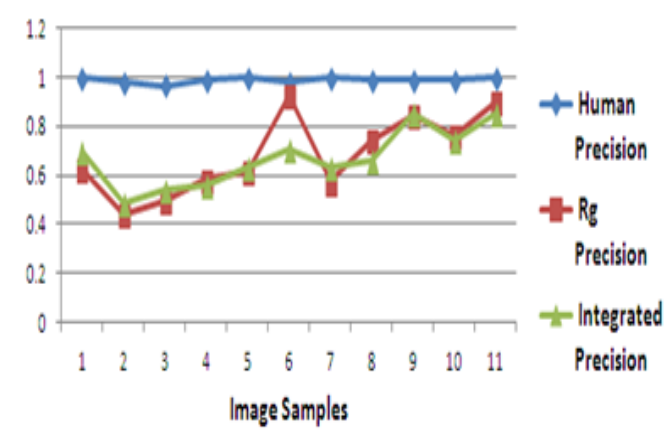

Figure 5. Comparison of the Precision Values between Human, Region Growing and Integrated Segmentation.

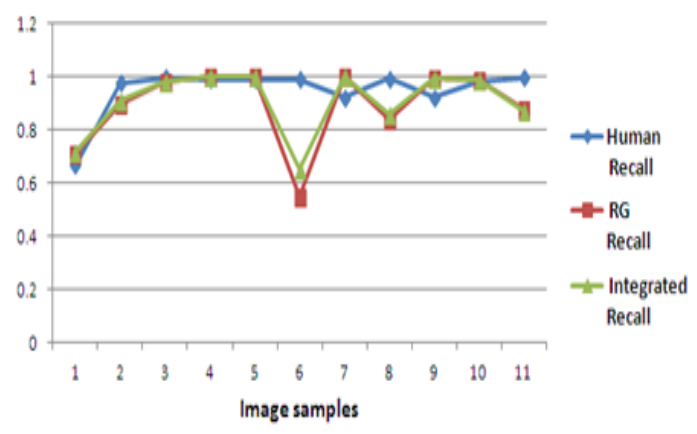

Figure 6. Comparison of Recall Values between Human, Region Growing and Integrated Segmentation 
Figures 4 and 5 depict that along the 11 tested images; the proposed integrated segmentation approach provided superior F-score and precision values; respectively compared to the region growing method. Moreover, Figure 6 illustrates the comparative results for the three methods in terms of the recall. In addition, Table 1 shows the overall comparative result of the 11 images.

Table 1. Comparative result analysis of the proposed work with human and region growing methods

\begin{tabular}{|c|c|c|c|c|c|c|c|c|c|}
\hline \multirow[t]{2}{*}{ Image } & \multicolumn{3}{|c|}{ Human } & \multicolumn{3}{|c|}{ Region Growing $(0.2)$} & \multicolumn{3}{|c|}{ Integrated } \\
\hline & F-Score & Recall & Precision & F-Score & Recall & Precision & F-Score & Recall & Precision \\
\hline 1 & 0.80 & 0.67 & 1 & 0.66 & 0.71 & 0.62 & 0.71 & 0.72 & 0.70 \\
\hline 3 & 0.98 & 0.99 & 0.97 & 0.66 & 0.98 & 0.50 & 0.70 & 0.98 & 0.54 \\
\hline 6 & 0.99 & 0.99 & 0.98 & 0.69 & 0.55 & 0.93 & 0.68 & 0.65 & 0.71 \\
\hline 7 & 0.96 & 0.92 & 1 & 0.73 & 1 & 0.57 & 0.77 & 1 & 0.63 \\
\hline 8 & 0.99 & 0.99 & 0.99 & 0.80 & 0.84 & 0.75 & 0.68 & 0.86 & 0.56 \\
\hline 9 & 0.96 & 0.92 & 0.99 & 0.91 & 0.99 & 0.84 & 0.91 & 1 & 0.83 \\
\hline 10 & 0.99 & 0.99 & 0.99 & 0.86 & 0.98 & 0.76 & 0.85 & 0.99 & 0.74 \\
\hline
\end{tabular}

The results in Figures 4 through 6 are tabulated in Table 1 to demonstrate a comparative study of the various metrics for the different methods compared to the proposed method. These results establish the following:

a. The proposed integrated system has higher recall value than that obtained using the region growing with a 0.2 threshold. Although, its recall value is comparable with that obtained by the human segmented images.

b. In terms of the precision, the proposed method is comparable to the region growing with 0.2 threshold method precision, while the human segmented images achievesthe best precision values.

c. The average values of the metrics prove the same conclusion, and clarifies that even the proposed method has superior averagerecall value than the region growing method, but it almost has the same precision and F-score values.

d. The proposed algorithm has a very higher recall than the region growing method, which ultimately reduces the precision performance as both the recall and precision are complementary metrics.

e. It is noticeable from the images under test that if the image has a dark background with bright object, the proposed method provides superior recall to that obtained by the ground truth human segmentation method as well as the region growing method. As in images numbers $(4,5,7$, and 9), where the proposed method gain recall of value 1 , which is outperforming the other methods.

A precision-recall curve is a parameterized curve that confines the trade-off between the accuracy and noise. Thus, Figures 7 and 8 indicatethe recall and precision comparison when using the region growing method and the proposed method as well; respectively. Furthermore, Figure 9 indicates the relation between the proposed method and the region growing in terms of the recall and precision.

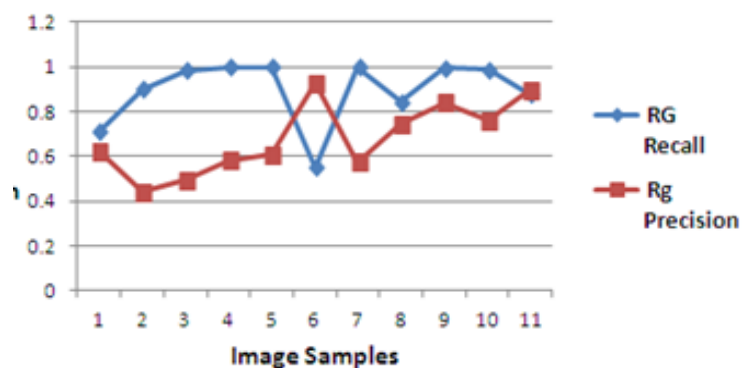

Figure 6. Recall and Precision Values Of Region Growing Segmentation Method

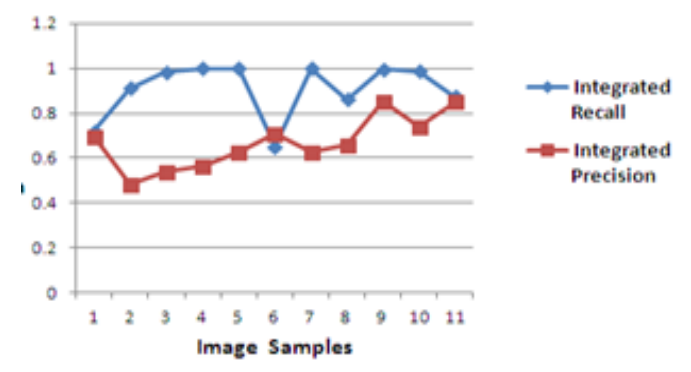

Figure 7. Recall and Precision Values of Integrated Segmentation Proposed Method 


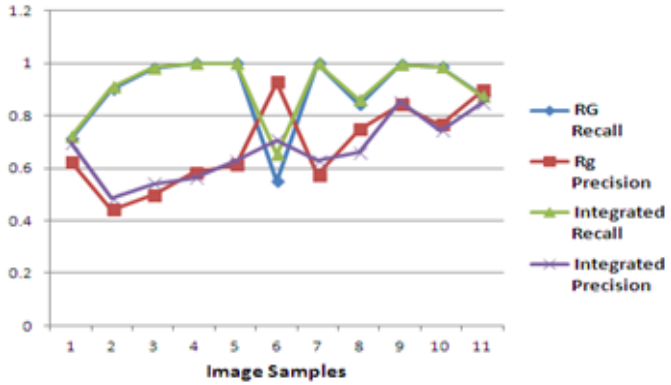

Figure 8. Recall and Precision Values Comparison between Region Growing and Integrated Segmentation Methods

Thus, Figures 7 through 9 establish the inverse relation between the two metrics while comparing the recall and precision of the region growing and the integrated segmentation proposed method; respectively.

Consequently, from the preceding results, it is established that the proposed system performance is almost matched with the same obtained using the ground truth results. It provides the best recall of ideal value equal one in the case of the dark background with bright objects. Since, segmentation has a significant role in several applications [21-30], Thus, it is recommended to apply the proposed method in the medical domain applications as a future work.

\section{CONCLUSION}

Segmentation is usually the first phase in any automated image analysis or interpretation. It bridges the gap between the low-level and high-level image processing. Severaltypes of segmentation technique can be applied in any application including the recognition, detection, and measurement of objects in images. Thresholding employs global information in the form of pixel similarities all over an image, while region growing applies local information. The threshold value can be decided through a variety of schemes such as manual selection, iterative method, Otsu's method, local thresholding.

In the proposed work, a novel integrated method is used to find the segmented region of interest of an image. It is considered as a hybrid system as it combines the work of thresholding with region growing. Also, it is an interactive system as the selection of the seed points is manual i.e. it is real time based on user area of interest. The evaluation of the performance of the proposed work is based on evaluation by different parameters (recall, precision, F-score). The results show the measures taken using the proposed method match with the qualitative analysis by visual inspection that are considered as an ideal edge in the ground truth. In the further work semi-automatic segmentation can be developed to identify fine blood vessels in magnetic resonance angiogram (MRA) and Computed tomography angiography (CTA) volume data.

\section{REFERENCES}

[1] A.R. Marcal, A. Rodrigues, and M. Cunha, "Evaluation of satellite image segmentation using synthetic images", 2010IEEE International Geoscience and Remote Sensing Symposium (IGARSS), pp. 2210-2213, 25-30 July 2010.

[2] R. Huang, and K. Ma, "RGVis: Region Growing Based Techniques for Volume Visualization", International Conference on Cognition \& Recognition, April 2008.

[3] S. Bose, A. Mukherjee, Madhulika, S. Chakraborty, S. Samanta, and N. Dey, "Parallel Image Segmentation using Multi-Threading and K-Means Algorithm”, 2013 IEEE International Conference on Computational Intelligence and Computing Research (ICCIC), Madurai, 26-28 Dec. 2013.

[4] W. Tao, H. Jin, and Y. Zhang, "Color Image Segmentation Based on Mean Shift and Normalized Cuts", IEEE Transactions on Systems, Man, and Cybernetics-Part B: Cybernetics, Vol. 37, No. 5, pp. 1382- 1389, October 2007.

[5] R.C. Gonzalez, and R.E. Woods, Digital Image Processing, Prentice-Hall, Englewood Cliffs, NJ, 2002.

[6] B. Georgescu, I. Shimshoni, and P. Meer, "Mean Shift Based Clustering in High Dimensions: A Texture Classification Example", Intl Conf on Computer Vision, 2003.

[7] V. Sivaram, P. Mirajkar, K. Deepak, V. Kishore, and G. Harikrishna, "Generic Medical-Image Region Segmentation using Gabor Filters", International Conference on Cognition and Recognition, 2008.

[8] S. Al-amri, N. Kalyankar, and S. Khamitkar, "Image Segmentation by Using Thershod Techniques", Journal of Computing, Vol. 2, No.5, May 2010. 
[9] B. Popescu, A. Iancu, M. Brezovan, and D.D. Burdescu, "Boundary-Based Measures for Evaluation of Color Image Segmentation", 2010 Second International Conferences on Advances in Multimedia (MMEDIA), pp. 162-167, 1319 June 2010.

[10] Y. Zhou, S. Jiang, and M. Yin, “A Region-Based Image Segmentation Method with Mean-Shift Clustering Algorithm", Fifth International Conference on Fuzzy Systems and Knowledge Discovery (FSKD '08.), Vol. 2, pp. 366, 370, 18-20 Oct. 2008.

[11] M. Kaas, A. Witkin, and D. Terzopoulos, "Snakes: Active contour models", International Journal of Computer Vision, Vol. 1, pp. 321 - 331, 1988.

[12] W. Yussof, and H. Burkhardt, "3D Volumetric CT Liver Segmentation Using Hybrid Segmentation Techniques", International Conference of Soft Computing and Pattern Recognition (SOCPAR '09), pp. 404-408, 47 Dec. 2009.

[13] Alamgir Nyma, Myeongsu Kang, Yung-Keun Kwon, Cheol-Hong Kim, and Jong-Myon Kim “A Hybrid Technique forMedical Image Segmentation", Article ID 830252, Journal of Biomedicine and Biotechnology Hindawi Publishing Corporation, Vol. 2012, 2012.

[14] D. Junfeng, and Y. Yunyang, "The fast Medical Image Segmentation of target Region based on Improved FM Algorithm", 2012 International Workshop of Information and Electronics Engineering (IWIEE), Vol. 29, pp. 48 $52,2012$.

[15] R. Adams, and L. Bischof, "Seeded region growing", IEEE Transactions on Pattern Analysis and Machine Intelligence, Vol. 16, pp. 641-647,1994.

[16] H. Rai, and T.R. Nair, "Gradient Based Seeded Region Grow method for CT Angiographic Image Segmentation", Inter JRI Computer Science and Networking, Vol. 1, No. 1, pp. 1-6, July 2009.

[17] S. Alpert, and M. Galun, R. Basri, and A. Brandt, "Image Segmentation by Probabilistic Bottom-Up Aggregation and Cue Integration", Proceedings of the IEEE Conference on Computer Vision and Pattern Recognition, June, 2007.

[18] R. Hn-RoldaHn, J. Hmez-Lopera, C. Atae-Allah, J. Hnez-Aroza, and P. Luque-Escamilla, “A measure of quality for evaluating methods of segmentation and edge detection", Pattern Recognition, Vol. 34, pp 969-980, 2001

[19] M. Buckland, and F. Gey "The relationship between Recall and Precision", Journal of the American Society for Information Science, Vol. 45, pp. 12-19, 1994.

[20] P. DMW, "Evaluation: From Precision, Recall and F-factor to ROC, Informedness, Markedness\& Correlation", Journal of Machine Learning Technologies, Vol. 2, pp. 37-63, 2007.

[21] T. Jyothirmayi, K.S. Rao, P.S. Rao, and C. Satyanarayana, "Image segmentation based on Doubly Truncated Generalized Laplace Mixture Model and K Means Clustering”, International Journal of Electrical and Computer Engineering (IJECE), Vol 6, No. 5, 2016.

[22] J. Harikiran, P.V. Lakshmi, and R.K. Kumar, "Multiple Feature Fuzzy c-means Clustering Algorithm for Segmentation of Microarray Images", International Journal of Electrical and Computer Engineering (IJECE), Vol. 5, No. 5, pp. 1045-1053, October 2015.

[23] Saparudin and G. Sulong, "Segmentation of Fingerprint Image Based on Gradient Magnitude and Coherence", International Journal of Electrical and Computer Engineering (IJECE), Vol. 5, No. 5, pp. 1202-1215, October 2015.

[24] N. Dey, A.B. Ro, M. Pal, and A. Das, "FCM Based Blood Vessel Segmentation Method for Retinal Images", International Journal of Computer Science and Network (IJCSN), Vol. 1, No. 3, June 2012.

[25] S. Samanta, N. Dey, P. Das, S. Acharjee, and S. S. Chaudhuri, "Multilevel Threshold Based Gray Scale Image Segmentation using Cuckoo Search", International Conference on Emerging Trends in Electrical, Communication and Information Technologies (ICECIT), Dec 12-23, 2012.

[26] P. Roy, S. Goswami, S. Chakraborty, A.T. Azar, and N. Dey, "Image Segmentation using Rough Set theory: A Review", International Journal of Rough Sets and Data Analysis, Vol. 1, No. 2, pp. 62-74, 2015.

[27] S. Samanta, S. Acharjee, A. Mukherjee, D. Das, and N. Dey, "Ant Weight Lifting Algorithm for Image Segmentation", 2013 IEEE International Conference on Computational Intelligence and Computing Research (ICCIC), Madurai, Dec 26-28 2013.

[28] S. Bose, A. Mukherjee, S. Chakraborty, S. Samanta, and N. Dey, "Parallel Image Segmentation using MultiThreading and K-Means Algorithm", 2013 IEEE International Conference on Computational Intelligence and Computing Research(ICCIC), Madurai, Dec 26-28 2013.

[29] G. Pal, S. Acharjee, D. Rudrapaul, A.S. Ashour, and N. Dey, "Video Segmentation using Minimum Ratio Similarity Measurement", International Journal of Image Mining, Vol 1, No. 1, 2015.

[30] R. Kumar, F.A. Talukdar, N. Dey, A.S. Ashour, V. Santhi, V.E. Balas, F. Shi, "Histogram Thresholding in Image Segmentation: A Joint Level Set Method and Lattice Boltzmann Method based Approach", 2015 International Conference on Information Technology and Intelligent Transportation Systems (ITITS 2015), Xi'an, China, 2015. 